\title{
Incomplete split-block designs constructed by affine $\alpha$-resolvable designs
}

\author{
Kazuhiro Ozawa ${ }^{1}$, Shinji Kuriki ${ }^{2}$, Stanisław Mejza ${ }^{3}$ \\ ${ }^{1}$ Department of Nursing, Gifu College of Nursing, Hashima, Gifu, 501-6295, Japan, \\ e-mail: ozawa@gifu-cn.ac.jp \\ ${ }^{2}$ Department of Mathematical Sciences, Graduate School of Engineering, Osaka \\ Prefecture University, Naka-ku, Sakai, Osaka, 599-8531, Japan, \\ e-mail: kuriki@ms.osakafu-u.ac.jp \\ ${ }^{3}$ Department of Mathematical and Statistical Methods, Poznań University of Life \\ Sciences, Wojska Polskiego 28, PL-60-637 Poznań, Poland, \\ e-mail: stanislaw.mejza@up.poznan.pl
}

\begin{abstract}
SUMMARY
We construct an incomplete split-block design (ISBD) by the semiKronecker product of two affine $\alpha$-resolvable designs for row and column treatments. We characterize such ISBDs with respect to the general balance property and we give the stratum efficiency factors for the ISBDs.

Key words: affine $\alpha$-resolvable designs, incomplete split-block designs, general balance property, stratum efficiency factors
\end{abstract}

\section{Introduction}

We consider a two-factor experiment of split-block type with $b$ blocks, in which the first factor $A$ occurs at $v_{1}$ levels $A_{1}, A_{2}, \ldots, A_{v_{1}}$ and the second factor $B$ occurs at $v_{2}$ levels $B_{1}, B_{2}, \ldots, B_{v_{2}}$. Each block is divided into $k_{1}$ rows and $k_{2}$ columns. The levels of $A$, called row treatments, are randomly assigned to the rows, and the levels of $B$, called column treatments, are randomly assigned to the columns. Such a design is called a split-block design. Here we consider an incomplete split-block design (ISBD) such that $k_{1}<v_{1}$ and $k_{2}<v_{2}$.

ISBDs are often used in the biological, agricultural and environmental sciences. Many authors (for example Mejza, 1987, 1998; Mejza, 1992 and Hering and Mejza, 1997) have considered methods for the analysis of data and constructions for ISBDs. In particular, Mejza (1992) and Hering and Mejza (1997) considered a mixed linear model with fixed treatment effects 
and random block, row and column effects. The $h$ th factorial treatment combination effect $\tau_{h}$ is defined by

$$
\tau_{h}=\mu+\alpha_{i}+\beta_{j}+(\alpha \beta)_{i j}
$$

for $h=(i-1) v_{2}+j, i=1,2, \ldots, v_{1}$ and $j=1,2, \ldots, v_{2}$, where $\mu$ is the general mean, $\alpha_{i}$ denotes the main effect of level $A_{i}$ of the factor $A, \beta_{j}$ denotes the main effect of level $B_{j}$ of the factor $B$, and $(\alpha \beta)_{i j}$ denotes the interaction effect of $A_{i}$ and $B_{j}$. Here $\sum_{i=1}^{v_{1}} \alpha_{i}=0, \sum_{j=1}^{v_{2}} \beta_{j}=0, \sum_{i=1}^{v_{1}}(\alpha \beta)_{i j}=0$ for $j=1,2, \ldots, v_{2}$ and $\sum_{j=1}^{v_{2}}(\alpha \beta)_{i j}=0$ for $i=1,2, \ldots, v_{1}$. This model (cf. Hering and Mejza, 1997) results from a three-step randomization, i.e., the randomization of blocks, the randomization of the rows (or columns) within each block and the randomization of the columns (or rows) within each block. This randomization leads to experiments with orthogonal block structure as defined by Nelder (1965a, 1965b), and the multistratum analysis proposed by Nelder (1965a, 1965b) and Houtman and Speed (1983) can be applied to the analysis of the experiments. In the case of ISBDs we have four strata, besides the zero stratum connected with the general mean only: (I) the inter-block stratum, (II) the inter-row (within blocks) stratum, (III) the inter-column (within blocks) stratum and (IV) the inter-plot stratum. The statistical properties of ISBDs are strictly connected with the eigenvalues and the eigenvectors of the stratum information matrices for the treatment combinations. Here we assume that every treatment combination $A_{i} B_{j}\left(i=1,2, \ldots, v_{1}, j=1,2, \ldots, v_{2}\right)$ occurs in precisely $r$ blocks, and that the treatment combinations are arranged in lexicographic order. The stratum information matrices are

$$
\begin{aligned}
& \mathbf{A}_{1}=\frac{1}{k_{1} k_{2}} \mathbf{N}_{1} \mathbf{N}_{1}^{\prime}-\frac{r}{v} \mathbf{J}_{v}, \quad \mathbf{A}_{2}=\frac{1}{k_{2}} \mathbf{N}_{2} \mathbf{N}_{2}^{\prime}-\frac{1}{k_{1} k_{2}} \mathbf{N}_{1} \mathbf{N}_{1}^{\prime}, \\
& \mathbf{A}_{3}=\frac{1}{k_{1}} \mathbf{N}_{3} \mathbf{N}_{3}^{\prime}-\frac{1}{k_{1} k_{1}} \mathbf{N}_{1} \mathbf{N}_{1}^{\prime} \text { and } \\
& \mathbf{A}_{4}=r \mathbf{I}_{v}-\frac{1}{k_{2}} \mathbf{N}_{2} \mathbf{N}_{2}^{\prime}-\frac{1}{k_{1}} \mathbf{N}_{3} \mathbf{N}_{3}^{\prime}+\frac{1}{k_{1} k_{2}} \mathbf{N}_{1} \mathbf{N}_{1}^{\prime},
\end{aligned}
$$

where $v=v_{1} v_{2}, \mathbf{N}_{1}, \mathbf{N}_{2}$ and $\mathbf{N}_{3}$ are the incidence matrices for treatment combinations vs. blocks, treatment combinations vs. rows and treatment combinations vs. columns, respectively, $\mathbf{I}_{v}$ is the identity matrix of order $v$ and $\mathbf{J}_{v}$ is a $v \times v$ matrix whose elements are all unity. The eigenvalues of a matrix $\mathbf{A}_{f}^{*}=r^{-1} \mathbf{A}_{f}$ can be identified as the stratum efficiency factors of the design for $f=1,2,3,4$ (see Houtman and Speed, 1983 and Mejza, 1992) and the corresponding eigenvectors define contrasts of treatment effects, which are called the basic contrasts (see Pearce et al., 1974). For details of the above argument, we refer to Mejza (1992) and Hering and Mejza (1997). 
Hering and Mejza $(1997,2002)$ considered the constructions of ISBDs by the Kronecker product of the incidence matrices of two designs. Ozawa et al. (2000) introduced balanced ISBDs and semi-balanced ISBDs, and they gave the necessary conditions for their existence and some methods for constructing them. Ozawa et al. (2002a, 2002b) characterized ISBDs with respect to their optimal properties and gave some methods for constructing optimal designs. ISBDs constructed by these methods usually need a large number of units. Mejza et al. (2001), Kuriki et al. (2005) and Mejza et al. (2009) considered constructions of ISBDs by a modified Kronecker product (called the semi-Kronecker product) of the incidence matrices of two resolvable designs. Since these designs have smaller numbers of blocks than the conventional split-block experiments, they would be useful in practice. In this paper, we construct an ISBD by the semi-Kronecker product of the incidence matrices of two affine $\alpha$-resolvable designs for row and column treatments, and these ISBDs also include those obtained by Mejza et al. (2001), Kuriki et al. (2005) and Mejza et al. (2009). We characterize such ISBDs with respect to the general balance property (see Houtman and Speed, 1983) and we give the stratum efficiency factors for the ISBDs.

\section{The method of construction of ISBDs}

Firstly, we need the semi-Kronecker product of two matrices, which will be used to construct ISBDs (see Mejza et al., 2001). Suppose that two matrices $\mathbf{C}$ and $\mathbf{D}$ are divided into the same number $t$ of submatrices as follows:

$$
\mathbf{C}=\left[\mathbf{C}_{1}: \mathbf{C}_{2}: \cdots: \mathbf{C}_{t}\right] \text { and } \mathbf{D}=\left[\mathbf{D}_{1}: \mathbf{D}_{2}: \cdots: \mathbf{D}_{t}\right]
$$

Then, the semi-Kronecker product $\mathbf{C} \tilde{\otimes} \mathbf{D}$ is given by

$$
\mathbf{C} \tilde{\otimes} \mathbf{D}=\left[\mathbf{C}_{1} \otimes \mathbf{D}_{1}: \mathbf{C}_{2} \otimes \mathbf{D}_{2}: \cdots: \mathbf{C}_{t} \otimes \mathbf{D}_{t}\right],
$$

where $\otimes$ denotes the usual Kronecker product. This type of Kronecker product was first considered by Khatri and Rao (1968).

Next, we need an affine $\alpha$-resolvable design. A design with $v$ treatments, $r$ replications of each treatment and $k$ plots within each block is denoted by $\mathrm{D}(v, r, k)$. If the collection of blocks of a $\mathrm{D}(v, r, k)$ can be partitioned into some classes (called resolution classes) such that every treatment occurs precisely $\alpha$ times in every class, the design is said to be $\alpha$-resolvable (resolvable for brevity, if $\alpha=1$ ) and it is denoted by $\alpha-\operatorname{RD}(v, r, k)$. The parameters of an $\alpha-\operatorname{RD}(v, r, k)$ satisfy the following conditions:

$$
\alpha v=k \beta, \quad b=t \beta \quad \text { and } \quad r=\alpha t,
$$


where $\beta$ is the number of blocks within each resolution class, $b$ is the total number of blocks and $t$ is the number of resolution classes. Moreover, an $\alpha$-resolvable design is called an affine $\alpha$-resolvable design if the number of common treatments containing two distinct blocks in the same resolution class is $q_{1}$, and the number of common treatments containing two distinct blocks belonging to different resolution classes is $q_{2}$. The parameters of an affine $\alpha-\operatorname{RD}(v, r, k)$ satisfy the following additional conditions:

$$
q_{1}=\frac{\alpha-1}{\beta-1} k, \quad q_{2}=\frac{k^{2}}{v}, \quad \beta>1 \quad \text { and } \quad k>q_{1} .
$$

Many authors have given constructions of affine $\alpha$-resolvable balanced and partially balanced incomplete block designs (see, for example, Kageyama 1973; Bailey et al., 1995; Caliński and Kageyama, 2003 and Kadowaki and Kageyama, 2009). By taking certain resolution classes of these designs, we can obtain the affine $\alpha$-resolvable designs considered above. In particular, an affine $\alpha$-resolvable design with $\alpha=1, q_{1}=0$ and $q_{2}=1$ is called a square lattice design.

We construct an ISBD from two affine $\alpha$-resolvable designs. Let

$$
\mathbf{N}_{A}=\left(\mathbf{N}_{A 1}: \mathbf{N}_{A 2}: \cdots: \mathbf{N}_{A t}\right) \quad \text { and } \quad \mathbf{N}_{B}=\left(\mathbf{N}_{B 1}: \mathbf{N}_{B 2}: \cdots: \mathbf{N}_{B t}\right)
$$

be the incidence matrices of an affine $\alpha_{1}-\operatorname{RD}\left(v_{1}, r_{1}, k_{1}\right)$ and an affine $\alpha_{2^{-}}$ $\mathrm{RD}\left(v_{2}, r_{2}, k_{2}\right)$ respectively, where the designs have the same number $t$ of resolution classes, and $\mathbf{N}_{A i}$ and $\mathbf{N}_{B i}$ correspond to the $i$ th of these resolution classes. By the definition of affine $\alpha$-resolvable designs,

$$
\begin{aligned}
& \mathbf{N}_{A i}^{\prime} \mathbf{N}_{A i}=\left(k_{1}-q_{11}\right) \mathbf{I}_{\beta_{1}}+q_{11} \mathbf{J}_{\beta_{1}}, \quad \mathbf{N}_{A i}^{\prime} \mathbf{N}_{A j}=q_{12} \mathbf{J}_{\beta_{1}}, \\
& \mathbf{N}_{B i}^{\prime} \mathbf{N}_{B i}=\left(k_{2}-q_{21}\right) \mathbf{I}_{\beta_{2}}+q_{21} \mathbf{J}_{\beta_{2}} \quad \text { and } \quad \mathbf{N}_{B i}^{\prime} \mathbf{N}_{B j}=q_{22} \mathbf{J}_{\beta_{2}}
\end{aligned}
$$

hold for $i, j=1,2, \ldots, t, i \neq j$, where $q_{11}=k_{1}\left(\alpha_{1}-1\right) /\left(\beta_{1}-1\right), q_{12}=k_{1}^{2} / v_{1}$, $\beta_{1}=\alpha_{1} v_{1} / k_{1}, q_{21}=k_{2}\left(\alpha_{2}-1\right) /\left(\beta_{2}-1\right), q_{22}=k_{2}^{2} / v_{2}$ and $\beta_{2}=\alpha_{2} v_{2} / k_{2}$. Now we construct an ISBD, say $\tilde{\mathcal{D}}$, such that its incidence matrix $\mathbf{N}_{1}$ is obtained by the semi-Kronecker product of $\mathbf{N}_{A}$ and $\mathbf{N}_{B}$, i.e.,

$$
\mathbf{N}_{1}=\mathbf{N}_{A} \tilde{\otimes} \mathbf{N}_{B}=\left(\mathbf{N}_{A 1} \otimes \mathbf{N}_{B 1}: \mathbf{N}_{A 2} \otimes \mathbf{N}_{B 2}: \cdots: \mathbf{N}_{A t} \otimes \mathbf{N}_{B t}\right)
$$

We use two affine $\alpha$-resolvable designs for row and column treatments. The ISBD $\tilde{\mathcal{D}}$ has $v_{1} v_{2}$ treatment combinations, $v_{1}$ row treatments, $v_{2}$ column 
treatments and $t \beta_{1} \beta_{2}$ blocks with $k_{1}$ rows and $k_{2}$ columns. For $\tilde{\mathcal{D}}$, we can express $\mathbf{N}_{2}$ and $\mathbf{N}_{3}$ as

$$
\mathbf{N}_{2}=\left(\mathbf{1}_{\alpha_{1}}^{\prime} \otimes \mathbf{I}_{v_{1}} \otimes \mathbf{N}_{B 1}: \mathbf{1}_{\alpha_{1}}^{\prime} \otimes \mathbf{I}_{v_{1}} \otimes \mathbf{N}_{B 2}: \cdots: \mathbf{1}_{\alpha_{1}}^{\prime} \otimes \mathbf{I}_{v_{1}} \otimes \mathbf{N}_{B t}\right)
$$

and

$$
\mathbf{N}_{3}=\left(\mathbf{1}_{\alpha_{2}}^{\prime} \otimes \mathbf{N}_{A 1} \otimes \mathbf{I}_{v_{2}}: \mathbf{1}_{\alpha_{2}}^{\prime} \otimes \mathbf{N}_{A 2} \otimes \mathbf{I}_{v_{2}}: \cdots: \mathbf{1}_{\alpha_{2}}^{\prime} \otimes \mathbf{N}_{A t} \otimes \mathbf{I}_{v_{2}}\right),(6)
$$

arranging the rows or columns of blocks in a suitable order, where $\mathbf{1}_{v}$ is a $v \times 1$ vector whose elements are all unity. Therefore, from (4)-(6), we have

$$
\begin{aligned}
& \mathbf{N}_{1} \mathbf{N}_{1}^{\prime}=\sum_{i=1}^{t}\left(\mathbf{N}_{A i} \mathbf{N}_{A i}^{\prime} \otimes \mathbf{N}_{B i} \mathbf{N}_{B i}^{\prime}\right), \\
& \mathbf{N}_{2} \mathbf{N}_{2}^{\prime}=\sum_{i=1}^{t}\left(\alpha_{1} \mathbf{I}_{v_{1}} \otimes \mathbf{N}_{B i} \mathbf{N}_{B i}^{\prime}\right)=\alpha_{1} \mathbf{I}_{v_{1}} \otimes \mathbf{N}_{B} \mathbf{N}_{B}^{\prime}
\end{aligned}
$$

and

$$
\mathbf{N}_{3} \mathbf{N}_{3}^{\prime}=\sum_{i=1}^{t}\left(\mathbf{N}_{A i} \mathbf{N}_{A i}^{\prime} \otimes \alpha_{2} \mathbf{I}_{v_{2}}\right)=\alpha_{2} \mathbf{N}_{A} \mathbf{N}_{A}^{\prime} \otimes \mathbf{I}_{v_{2}}
$$

Note that $\tilde{\mathcal{D}}$ is also an $\alpha$-resolvable design with $\alpha=\alpha_{1} \alpha_{2}$ if the treatment combinations are regarded as the usual treatments.

Example 1. We consider an affine 2-RD $(9,4,6)$ and an affine $3-\mathrm{RD}(16,6,12)$ with $\beta_{1}=3, \beta_{2}=4, b_{1}=6, b_{2}=8, t=2, q_{11}=3, q_{12}=4, q_{21}=8, q_{22}=9$ and the incidence matrices $\mathbf{N}_{A}=\left(\mathbf{N}_{A 1}: \mathbf{N}_{A 2}\right)$ and $\mathbf{N}_{B}=\left(\mathbf{N}_{B 1}: \mathbf{N}_{B 2}\right)$, where

$$
\mathbf{N}_{A 1}=\left(\begin{array}{ccc}
1 & 0 & 1 \\
1 & 0 & 1 \\
1 & 0 & 1 \\
1 & 1 & 0 \\
1 & 1 & 0 \\
1 & 1 & 0 \\
0 & 1 & 1 \\
0 & 1 & 1 \\
0 & 1 & 1
\end{array}\right), \quad \mathbf{N}_{A 2}=\left(\begin{array}{ccc}
1 & 0 & 1 \\
1 & 1 & 0 \\
0 & 1 & 1 \\
1 & 0 & 1 \\
1 & 1 & 0 \\
0 & 1 & 1 \\
1 & 0 & 1 \\
1 & 1 & 0 \\
0 & 1 & 1
\end{array}\right)
$$




$$
\mathbf{N}_{B 1}=\left(\begin{array}{cccc}
1 & 1 & 1 & 0 \\
1 & 1 & 1 & 0 \\
1 & 1 & 1 & 0 \\
1 & 1 & 1 & 0 \\
1 & 1 & 0 & 1 \\
1 & 1 & 0 & 1 \\
1 & 1 & 0 & 1 \\
1 & 1 & 0 & 1 \\
1 & 0 & 1 & 1 \\
1 & 0 & 1 & 1 \\
1 & 0 & 1 & 1 \\
1 & 0 & 1 & 1 \\
0 & 1 & 1 & 1 \\
0 & 1 & 1 & 1 \\
0 & 1 & 1 & 1 \\
0 & 1 & 1 & 1
\end{array}\right) \quad \text { and } \quad \mathbf{N}_{B 2}=\left(\begin{array}{cccc}
1 & 1 & 1 & 0 \\
1 & 1 & 0 & 1 \\
1 & 0 & 1 & 1 \\
0 & 1 & 1 & 1 \\
1 & 1 & 1 & 0 \\
1 & 1 & 0 & 1 \\
1 & 0 & 1 & 1 \\
0 & 1 & 1 & 1 \\
1 & 1 & 1 & 0 \\
1 & 1 & 0 & 1 \\
1 & 0 & 1 & 1 \\
0 & 1 & 1 & 1 \\
1 & 1 & 1 & 0 \\
1 & 1 & 0 & 1 \\
1 & 0 & 1 & 1 \\
0 & 1 & 1 & 1
\end{array}\right)
$$

The ISBD $\tilde{\mathcal{D}}$ constructed by the semi-Kronecker product has 24 blocks with 6 rows and 12 columns in each block. The ISBD can be expressed in the following way:

$\left\{A_{1}, A_{2}, A_{3}, A_{4}, A_{5}, A_{6} \mid B_{1}, B_{2}, B_{3}, B_{4}, B_{5}, B_{6}, B_{7}, B_{8}, B_{9}, B_{10}, B_{11}, B_{12}\right\}$, $\left\{A_{1}, A_{2}, A_{3}, A_{4}, A_{5}, A_{6} \mid B_{1}, B_{2}, B_{3}, B_{4}, B_{5}, B_{6}, B_{7}, B_{8}, B_{13}, B_{14}, B_{15}, B_{16}\right\}$, $\left\{A_{1}, A_{2}, A_{3}, A_{4}, A_{5}, A_{6} \mid B_{1}, B_{2}, B_{3}, B_{4}, B_{9}, B_{10}, B_{11}, B_{12}, B_{13}, B_{14}, B_{15}, B_{16}\right\}$, $\left\{A_{1}, A_{2}, A_{3}, A_{4}, A_{5}, A_{6} \mid B_{5}, B_{6}, B_{7}, B_{8}, B_{9}, B_{10}, B_{11}, B_{12}, B_{13}, B_{14}, B_{15}, B_{16}\right\}$, $\left\{A_{4}, A_{5}, A_{6}, A_{7}, A_{8}, A_{9} \mid B_{1}, B_{2}, B_{3}, B_{4}, B_{5}, B_{6}, B_{7}, B_{8}, B_{9}, B_{10}, B_{11}, B_{12}\right\}$, $\left\{A_{4}, A_{5}, A_{6}, A_{7}, A_{8}, A_{9} \mid B_{1}, B_{2}, B_{3}, B_{4}, B_{5}, B_{6}, B_{7}, B_{8}, B_{13}, B_{14}, B_{15}, B_{16}\right\}$, $\left\{A_{4}, A_{5}, A_{6}, A_{7}, A_{8}, A_{9} \mid B_{1}, B_{2}, B_{3}, B_{4}, B_{9}, B_{10}, B_{11}, B_{12}, B_{13}, B_{14}, B_{15}, B_{16}\right\}$, $\left\{A_{4}, A_{5}, A_{6}, A_{7}, A_{8}, A_{9} \mid B_{5}, B_{6}, B_{7}, B_{8}, B_{9}, B_{10}, B_{11}, B_{12}, B_{13}, B_{14}, B_{15}, B_{16}\right\}$, $\left\{A_{1}, A_{2}, A_{3}, A_{7}, A_{8}, A_{9} \mid B_{1}, B_{2}, B_{3}, B_{4}, B_{5}, B_{6}, B_{7}, B_{8}, B_{9}, B_{10}, B_{11}, B_{12}\right\}$, $\left\{A_{1}, A_{2}, A_{3}, A_{7}, A_{8}, A_{9} \mid B_{1}, B_{2}, B_{3}, B_{4}, B_{5}, B_{6}, B_{7}, B_{8}, B_{13}, B_{14}, B_{15}, B_{16}\right\}$, $\left\{A_{1}, A_{2}, A_{3}, A_{7}, A_{8}, A_{9} \mid B_{1}, B_{2}, B_{3}, B_{4}, B_{9}, B_{10}, B_{11}, B_{12}, B_{13}, B_{14}, B_{15}, B_{16}\right\}$, $\left\{A_{1}, A_{2}, A_{3}, A_{7}, A_{8}, A_{9} \mid B_{5}, B_{6}, B_{7}, B_{8}, B_{9}, B_{10}, B_{11}, B_{12}, B_{13}, B_{14}, B_{15}, B_{16}\right\}$, $\left\{A_{1}, A_{2}, A_{4}, A_{5}, A_{7}, A_{8} \mid B_{1}, B_{2}, B_{3}, B_{5}, B_{6}, B_{7}, B_{9}, B_{10}, B_{11}, B_{13}, B_{14}, B_{15}\right\}$, $\left\{A_{1}, A_{2}, A_{4}, A_{5}, A_{7}, A_{8} \mid B_{1}, B_{2}, B_{4}, B_{5}, B_{6}, B_{8}, B_{9}, B_{10}, B_{12}, B_{13}, B_{14}, B_{16}\right\}$, $\left\{A_{1}, A_{2}, A_{4}, A_{5}, A_{7}, A_{8} \mid B_{1}, B_{3}, B_{4}, B_{5}, B_{7}, B_{8}, B_{9}, B_{11}, B_{12}, B_{13}, B_{15}, B_{16}\right\}$, $\left\{A_{1}, A_{2}, A_{4}, A_{5}, A_{7}, A_{8} \mid B_{2}, B_{3}, B_{4}, B_{6}, B_{7}, B_{8}, B_{10}, B_{11}, B_{12}, B_{14}, B_{15}, B_{16}\right\}$, $\left\{A_{2}, A_{3}, A_{5}, A_{6}, A_{8}, A_{9} \mid B_{1}, B_{2}, B_{3}, B_{5}, B_{6}, B_{7}, B_{9}, B_{10}, B_{11}, B_{13}, B_{14}, B_{15}\right\}$, $\left\{A_{2}, A_{3}, A_{5}, A_{6}, A_{8}, A_{9} \mid B_{1}, B_{2}, B_{4}, B_{5}, B_{6}, B_{8}, B_{9}, B_{10}, B_{12}, B_{13}, B_{14}, B_{16}\right\}$, 
$\left\{A_{2}, A_{3}, A_{5}, A_{6}, A_{8}, A_{9} \mid B_{1}, B_{3}, B_{4}, B_{5}, B_{7}, B_{8}, B_{9}, B_{11}, B_{12}, B_{13}, B_{15}, B_{16}\right\}$, $\left\{A_{2}, A_{3}, A_{5}, A_{6}, A_{8}, A_{9} \mid B_{2}, B_{3}, B_{4}, B_{6}, B_{7}, B_{8}, B_{10}, B_{11}, B_{12}, B_{14}, B_{15}, B_{16}\right\}$, $\left\{A_{1}, A_{3}, A_{4}, A_{6}, A_{7}, A_{9} \mid B_{1}, B_{2}, B_{3}, B_{5}, B_{6}, B_{7}, B_{9}, B_{10}, B_{11}, B_{13}, B_{14}, B_{15}\right\}$, $\left\{A_{1}, A_{3}, A_{4}, A_{6}, A_{7}, A_{9} \mid B_{1}, B_{2}, B_{4}, B_{5}, B_{6}, B_{8}, B_{9}, B_{10}, B_{12}, B_{13}, B_{14}, B_{16}\right\}$, $\left\{A_{1}, A_{3}, A_{4}, A_{6}, A_{7}, A_{9} \mid B_{1}, B_{3}, B_{4}, B_{5}, B_{7}, B_{8}, B_{9}, B_{11}, B_{12}, B_{13}, B_{15}, B_{16}\right\}$, $\left\{A_{1}, A_{3}, A_{4}, A_{6}, A_{7}, A_{9} \mid B_{2}, B_{3}, B_{4}, B_{6}, B_{7}, B_{8}, B_{10}, B_{11}, B_{12}, B_{14}, B_{15}, B_{16}\right\}$,

where $\left\{A_{i_{1}}, A_{i_{2}}, A_{i_{3}}, A_{i_{4}}, A_{i_{5}}, A_{i_{6}} \mid B_{j_{1}}, B_{j_{2}}, B_{j_{3}}, B_{j_{4}}, B_{j_{5}}, B_{j_{6}}, B_{j_{7}}, B_{j_{8}}, B_{j_{9}}\right.$, $\left.B_{j_{10}}, B_{j_{11}}, B_{j_{12}}\right\}$ denotes a block such that $A_{i_{1}}, A_{i_{2}}, A_{i_{3}}, A_{i_{4}}, A_{i_{5}}, A_{i_{6}}$ are row treatments and $B_{j_{1}}, B_{j_{2}}, B_{j_{3}}, B_{j_{4}}, B_{j_{5}}, B_{j_{6}}, B_{j_{7}}, B_{j_{8}}, B_{j_{9}}, B_{j_{10}}, B_{j_{11}}, B_{j_{12}}$ are column treatments. We note that if the ISBD is constructed by the usual Kronecker product of the incidence matrices, then the number of blocks becomes 48 . Generally, the number of blocks of an ISBD constructed by the Kronecker product is $t$ times larger than in the case of an ISBD constructed by the semi-Kronecker product.

\section{Stratum efficiency factors for the ISBD $\tilde{\mathcal{D}}$}

In this section, we give the stratum efficiency factors for the ISBD $\tilde{\mathcal{D}}$ constructed by the semi-Kronecker product of two affine $\alpha$-resolvable designs. To find the stratum efficiency factors, we need the eigenvalues of the stratum information matrices $\mathbf{A}_{1}, \mathbf{A}_{2}, \mathbf{A}_{3}$ and $\mathbf{A}_{4}$ of $\tilde{\mathcal{D}}$. If the stratum information matrices $\mathbf{A}_{1}, \mathbf{A}_{2}, \mathbf{A}_{3}$ and $\mathbf{A}_{4}$ have common eigenvectors, i.e., if $\tilde{\mathcal{D}}$ is generally balanced, then we can easily find the eigenvalues of the matrices. From (2) and (3),

$$
\mathbf{N}_{A i} \mathbf{N}_{A i}^{\prime} \mathbf{N}_{A j} \mathbf{N}_{A j}^{\prime}=\alpha_{1}^{2} q_{12} \mathbf{J}_{v_{1}} \quad \text { and } \quad \mathbf{N}_{B i} \mathbf{N}_{B i}^{\prime} \mathbf{N}_{B j} \mathbf{N}_{B j}^{\prime}=\alpha_{2}^{2} q_{22} \mathbf{J}_{v_{2}}(10)
$$

hold for $i, j=1,2, \ldots, t, i \neq j$. From (7)-(10), we see that the concurrence matrices $\mathbf{N}_{1} \mathbf{N}_{1}^{\prime}, \mathbf{N}_{2} \mathbf{N}_{2}^{\prime}$ and $\mathbf{N}_{3} \mathbf{N}_{3}^{\prime}$ are mutually commutative, which implies, from (1), that the stratum information matrices $\mathbf{A}_{1}, \mathbf{A}_{2}, \mathbf{A}_{3}$ and $\mathbf{A}_{4}$ are also mutually commutative. Therefore $\tilde{\mathcal{D}}$ is generally balanced.

We consider the eigenvectors of the concurrence matrices of the affine $\alpha_{1}-\mathrm{RD}\left(v_{1}, r_{1}, k_{1}\right)$ and the affine $\alpha_{2}-\mathrm{RD}\left(v_{2}, r_{2}, k_{2}\right)$ to find the common eigenvectors of $\mathbf{N}_{1} \mathbf{N}_{1}^{\prime}, \mathbf{N}_{2} \mathbf{N}_{2}^{\prime}$ and $\mathbf{N}_{3} \mathbf{N}_{3}^{\prime}$.

For the incidence matrix of the affine $\alpha_{1}-\mathrm{RD}\left(v_{1}, r_{1}, k_{1}\right)$, from (2), $\mathbf{N}_{A i} \mathbf{N}_{A i}^{\prime}$ has the eigenvalues $\alpha_{1} k_{1}, k_{1}-q_{11}$ and 0 , with multiplicities $1, \beta_{1}-1$ and $v_{1}-$ $\beta_{1}$ respectively, for each $i=1,2, \ldots, t$. From (10), $\mathbf{N}_{A 1} \mathbf{N}_{A 1}^{\prime}, \mathbf{N}_{A 2} \mathbf{N}_{A 2}^{\prime}, \ldots$, $\mathbf{N}_{A t} \mathbf{N}_{A t}^{\prime}$ are mutually commutative, so these concurrence matrices have common eigenvectors. Let $\mathbf{Q}_{\beta_{1}}=\left(\boldsymbol{q}_{0}^{\left(\beta_{1}\right)}, \boldsymbol{q}_{1}^{\left(\beta_{1}\right)}, \ldots, \boldsymbol{q}_{\beta_{1}-1}^{\left(\beta_{1}\right)}\right)$ be an orthogonal 
matrix of order $\beta_{1}$ with $\boldsymbol{q}_{0}^{\left(\beta_{1}\right)}=\frac{1}{\sqrt{\beta_{1}}} \mathbf{1}_{\beta_{1}}$. For each $i$ th resolution class, from (2), the mutually orthonormal eigenvectors of $\mathbf{N}_{A i} \mathbf{N}_{A i}^{\prime}$ corresponding to the eigenvalues $\alpha_{1} k_{1}$ and $k_{1}-q_{11}$ are given by

$$
\boldsymbol{x}_{i 0}=\frac{1}{\sqrt{\alpha_{1} k_{1}}} \mathbf{N}_{A i} \boldsymbol{q}_{0}^{\left(\beta_{1}\right)} \quad \text { and } \quad \boldsymbol{x}_{i j}=\frac{1}{\sqrt{k_{1}-q_{11}}} \mathbf{N}_{A i} \boldsymbol{q}_{j}^{\left(\beta_{1}\right)}
$$

for $j=1,2, \ldots, \beta_{1}-1$ respectively. In particular, $\boldsymbol{x}_{i 0}=\frac{1}{\sqrt{v_{1}}} \mathbf{1}_{v_{1}}$. From (2), the eigenvectors $\boldsymbol{x}_{i j}\left(j=0,1, \ldots, \beta_{1}-1\right)$ are also the eigenvectors of the concurrence matrix $\mathbf{N}_{A h} \mathbf{N}_{A h}^{\prime}(h \neq i)$ for the other resolution class, and the eigenvalues of $\mathbf{N}_{A h} \mathbf{N}_{A h}^{\prime}$ corresponding to $\boldsymbol{x}_{i 0}$ and $\boldsymbol{x}_{i j}(j \neq 0)$ are $\alpha_{1} k_{1}$ and 0 respectively. The mutually orthonormal common eigenvectors of $\mathbf{N}_{A 1} \mathbf{N}_{A 1}^{\prime}, \mathbf{N}_{A 2} \mathbf{N}_{A 2}^{\prime}, \ldots, \mathbf{N}_{A t} \mathbf{N}_{A t}^{\prime}$ corresponding to the eigenvalue 0 are denoted by $\boldsymbol{x}_{j}^{*}$ for $j=1,2, \ldots, v_{1}-t\left(\beta_{1}-1\right)-1$. These eigenvalues and eigenvectors are summarized in Table 1.

Similarly, we have the eigenvalues and the common eigenvectors of the concurrence matrices $\mathbf{N}_{B 1} \mathbf{N}_{B 1}^{\prime}, \mathbf{N}_{B 2} \mathbf{N}_{B 2}^{\prime}, \ldots, \mathbf{N}_{B t} \mathbf{N}_{B t}^{\prime}$ in the affine $\alpha_{2}-\mathrm{RD}\left(v_{2}, r_{2}, k_{2}\right)$ (see Table 2 ).

Here

$$
\boldsymbol{y}_{i 0}=\frac{1}{\sqrt{\alpha_{2} k_{2}}} \mathbf{N}_{B i} \boldsymbol{q}_{0}^{\left(\beta_{2}\right)} \quad \text { and } \quad \boldsymbol{y}_{i j}=\frac{1}{\sqrt{k_{2}-q_{21}}} \mathbf{N}_{B i} \boldsymbol{q}_{j}^{\left(\beta_{2}\right)}
$$

for $i=1,2, \ldots, t$ and $j=0,1, \ldots, \beta_{2}-1$, using an orthogonal matrix $\mathbf{Q}_{\beta_{2}}=\left(\boldsymbol{q}_{0}^{\left(\beta_{2}\right)}, \boldsymbol{q}_{1}^{\left(\beta_{2}\right)}, \ldots, \boldsymbol{q}_{\beta_{2}-1}^{\left(\beta_{2}\right)}\right)$ of order $\beta_{2}$ with $\boldsymbol{q}_{0}^{\left(\beta_{2}\right)}=\frac{1}{\sqrt{\beta_{2}}} \mathbf{1}_{\beta_{2}}$. The mutually orthonormal common eigenvectors of $\mathbf{N}_{B 1} \mathbf{N}_{B 1}^{\prime}, \mathbf{N}_{B 2} \mathbf{N}_{B 2}^{\prime}, \ldots, \mathbf{N}_{B t} \mathbf{N}_{B t}^{\prime}$ corresponding to the eigenvalue 0 are denoted by $\boldsymbol{y}_{j}^{*}$ for $j=1,2, \ldots, v_{2}-$ $t\left(\beta_{2}-1\right)-1$. The common eigenvectors in Table 1 and Table 2 are mutually orthogonal and play an important role in the succeeding argument.

Combining the above eigenvectors, we consider 10 sets of vectors as follows:

(1) $\frac{1}{\sqrt{v_{1}}} \mathbf{1}_{v_{1}} \otimes \frac{1}{\sqrt{v_{2}}} \mathbf{1}_{v_{2}}$,

(2) $\boldsymbol{x}_{i j} \otimes \frac{1}{\sqrt{v_{2}}} \mathbf{1}_{v_{2}}\left(i=1,2, \ldots, t, j=1,2, \ldots, m_{1}\right)$,

(3) $\boldsymbol{x}_{j}^{*} \otimes \frac{1}{\sqrt{v_{2}}} \mathbf{1}_{v_{2}}\left(j=1,2, \ldots, n_{1}\right)$, 
(4) $\frac{1}{\sqrt{v_{1}}} \mathbf{1}_{v_{1}} \otimes \boldsymbol{y}_{i j}\left(i=1,2, \ldots, t, j=1,2, \ldots, m_{2}\right)$,

(5) $\frac{1}{\sqrt{v_{1}}} \mathbf{1}_{v_{1}} \otimes \boldsymbol{y}_{j}^{*}\left(j=1,2, \ldots, n_{2}\right)$,

(6) $\boldsymbol{x}_{i j} \otimes \boldsymbol{y}_{i j^{\prime}}\left(i=1,2, \ldots, t, j=1,2, \ldots, m_{1}, j^{\prime}=1,2, \ldots, m_{2}\right)$,

(7) $\boldsymbol{x}_{i j} \otimes \boldsymbol{y}_{i^{\prime} j^{\prime}}\left(i, i^{\prime}=1, \ldots, t, i \neq i^{\prime}, j=1,2, \ldots, m_{1}, j^{\prime}=1, \ldots, m_{2}\right)$,

(8) $\boldsymbol{x}_{j}^{*} \otimes \boldsymbol{y}_{i j^{\prime}}\left(i=1,2, \ldots, t, j=1,2, \ldots, n_{1}, j^{\prime}=1,2, \ldots, m_{2}\right)$,

(9) $\boldsymbol{x}_{i j} \otimes \boldsymbol{y}_{j^{\prime}}^{*}\left(i=1,2, \ldots, t, j=1,2, \ldots, m_{1}, j^{\prime}=1,2, \ldots, n_{2}\right)$,

(10) $\boldsymbol{x}_{j}^{*} \otimes \boldsymbol{y}_{j^{\prime}}^{*}\left(j=1,2, \ldots, n_{1}, j^{\prime}=1,2, \ldots, n_{2}\right)$,

where $m_{1}=\beta_{1}-1, n_{1}=v_{1}-t\left(\beta_{1}-1\right)-1, m_{2}=\beta_{2}-1$ and $n_{2}=$ $v_{2}-t\left(\beta_{2}-1\right)-1$. It can easily be checked that the vectors of (1)-(10) are mutually orthonormal and that the total number of vectors is $v_{1} v_{2}$. We show that the vectors of (1)-(10) are the common eigenvectors of $\mathbf{N}_{1} \mathbf{N}_{1}^{\prime}, \mathbf{N}_{2} \mathbf{N}_{2}^{\prime}$ and $\mathbf{N}_{3} \mathbf{N}_{3}^{\prime}$ and we find the corresponding eigenvalues of $\mathbf{N}_{1} \mathbf{N}_{1}^{\prime}, \mathbf{N}_{2} \mathbf{N}_{2}^{\prime}$ and $\mathbf{N}_{3} \mathbf{N}_{3}^{\prime}$.

Table 1. Eigenvalues and common eigenvectors of $\mathbf{N}_{A i} \mathbf{N}_{A i}^{\prime}$ in the affine $\alpha_{1}-\mathrm{RD}\left(v_{1}, r_{1}, k_{1}\right)$

\begin{tabular}{|c|c|c|c|c|}
\hline \multicolumn{4}{|c|}{ Eigenvalues } & \multirow[b]{2}{*}{ Common eigenvectors } \\
\hline $\mathbf{N}_{A 1} \mathbf{N}_{A 1}^{\prime}$ & $\mathbf{N}_{A 2} \mathbf{N}_{A 2}^{\prime}$ & $\cdots$ & $\mathbf{N}_{A t} \mathbf{N}_{A t}^{\prime}$ & \\
\hline$\alpha_{1} k_{1}$ & $\alpha_{1} k_{1}$ & $\cdots$ & $\alpha_{1} k_{1}$ & $\boldsymbol{x}_{i 0}=\frac{1}{\sqrt{v_{1}}} \mathbf{1}_{v_{1}}$ \\
\hline$k_{1}-q_{11}$ & 0 & $\cdots$ & 0 & $\boldsymbol{x}_{1 j}\left(j=1,2, \ldots, \beta_{1}-1\right)$ \\
\hline 0 & $k_{1}-q_{11}$ & $\cdots$ & 0 & $\boldsymbol{x}_{2 j}\left(j=1,2, \ldots, \beta_{1}-1\right)$ \\
\hline$\vdots$ & $\vdots$ & $\vdots$ & $\vdots$ & $\vdots$ \\
\hline 0 & 0 & $\cdots$ & $k_{1}-q_{11}$ & $\boldsymbol{x}_{t j}\left(j=1,2, \ldots, \beta_{1}-1\right)$ \\
\hline 0 & 0 & $\cdots$ & 0 & $\boldsymbol{x}_{j}^{*}\left(j=1,2, \ldots, v_{1}-t\left(\beta_{1}-1\right)-1\right)$ \\
\hline
\end{tabular}

Table 2. Eigenvalues and common eigenvectors of $\mathbf{N}_{B i} \mathbf{N}_{B i}^{\prime}$ in the affine $\alpha_{2}-\mathrm{RD}\left(v_{2}, r_{2}, k_{2}\right)$

\begin{tabular}{|c|c|c|c|c|}
\hline \multicolumn{4}{|c|}{ Eigenvalues } & \multirow{2}{*}{ Common eigenvectors } \\
\hline $\mathbf{N}_{B 1} \mathbf{N}_{B 1}^{\prime}$ & $\mathbf{N}_{B 2} \mathbf{N}_{B 2}^{\prime}$ & $\cdots$ & $\mathbf{N}_{B t} \mathbf{N}_{B t}^{\prime}$ & \\
\hline$\alpha_{2} k_{2}$ & $\alpha_{2} k_{2}$ & $\cdots$ & $\alpha_{2} k_{2}$ & $\boldsymbol{y}_{i 0}=\frac{1}{\sqrt{v_{2}}} \mathbf{1}_{v_{2}}$ \\
\hline$k_{2}-q_{21}$ & 0 & $\cdots$ & 0 & $\boldsymbol{y}_{1 j}\left(j=1,2, \ldots, \beta_{2}-1\right)$ \\
\hline 0 & $k_{2}-q_{21}$ & $\cdots$ & 0 & $\boldsymbol{y}_{2 j}\left(j=1,2, \ldots, \beta_{2}-1\right)$ \\
\hline$\vdots$ & $\vdots$ & $\vdots$ & $\vdots$ & $\vdots$ \\
\hline 0 & 0 & $\cdots$ & $k_{2}-q_{21}$ & $\boldsymbol{y}_{t j}\left(j=1,2, \ldots, \beta_{2}-1\right)$ \\
\hline 0 & 0 & $\cdots$ & 0 & $\boldsymbol{y}_{j}^{*}\left(j=1,2, \ldots, v_{2}-t\left(\beta_{2}-1\right)-1\right)$ \\
\hline
\end{tabular}


Firstly, we consider the matrix $\mathbf{N}_{1} \mathbf{N}_{1}^{\prime}$. For (1), we have, from (7), Table 1 and Table 2,

$$
\begin{aligned}
\mathbf{N}_{1} \mathbf{N}_{1}^{\prime}\left(\frac{1}{\sqrt{v_{1}}} \mathbf{1}_{v_{1}} \otimes \frac{1}{\sqrt{v_{2}}} \mathbf{1}_{v_{2}}\right) & =\sum_{i=1}^{t}\left(\mathbf{N}_{A i} \mathbf{N}_{A i}^{\prime} \otimes \mathbf{N}_{B i} \mathbf{N}_{B i}^{\prime}\right)\left(\frac{1}{\sqrt{v_{1}}} \mathbf{1}_{v_{1}} \otimes \frac{1}{\sqrt{v_{2}}} \mathbf{1}_{v_{2}}\right) \\
& =\sum_{i=1}^{t}\left(\mathbf{N}_{A i} \mathbf{N}_{A i}^{\prime} \frac{1}{\sqrt{v_{1}}} \mathbf{1}_{v_{1}}\right) \otimes\left(\mathbf{N}_{B i} \mathbf{N}_{B i}^{\prime} \frac{1}{\sqrt{v_{2}}} \mathbf{1}_{v_{2}}\right) \\
& =t \alpha_{1} k_{1} \alpha_{2} k_{2}\left(\frac{1}{\sqrt{v_{1}}} \mathbf{1}_{v_{1}} \otimes \frac{1}{\sqrt{v_{2}}} \mathbf{1}_{v_{2}}\right) .
\end{aligned}
$$

The corresponding eigenvalue is $t \alpha_{1} k_{1} \alpha_{2} k_{2}$.

For (2), we have

$$
\begin{aligned}
\mathbf{N}_{1} \mathbf{N}_{1}^{\prime}\left(\boldsymbol{x}_{i j} \otimes \frac{1}{\sqrt{v_{2}}} \mathbf{1}_{v_{2}}\right) & =\sum_{s=1}^{t}\left(\mathbf{N}_{A s} \mathbf{N}_{A s}^{\prime} \otimes \mathbf{N}_{B s} \mathbf{N}_{B s}^{\prime}\right)\left(\boldsymbol{x}_{i j} \otimes \frac{1}{\sqrt{v_{2}}} \mathbf{1}_{v_{2}}\right) \\
& =\sum_{s=1}^{t}\left(\mathbf{N}_{A s} \mathbf{N}_{A s}^{\prime} \boldsymbol{x}_{i j}\right) \otimes\left(\mathbf{N}_{B s} \mathbf{N}_{B s}^{\prime} \frac{1}{\sqrt{v_{2}}} \mathbf{1}_{v_{2}}\right) \\
& =\left(k_{1}-q_{11}\right) \alpha_{2} k_{2}\left(\boldsymbol{x}_{i j} \otimes \frac{1}{\sqrt{v_{2}}} \mathbf{1}_{v_{2}}\right) .
\end{aligned}
$$

The corresponding eigenvalue is $\left(k_{1}-q_{11}\right) \alpha_{2} k_{2}$.

For (4), we have

$$
\begin{aligned}
\mathbf{N}_{1} \mathbf{N}_{1}^{\prime}\left(\frac{1}{\sqrt{v_{1}}} \mathbf{1}_{v_{1}} \otimes \boldsymbol{y}_{i j}\right) & =\sum_{s=1}^{t}\left(\mathbf{N}_{A s} \mathbf{N}_{A s}^{\prime} \otimes \mathbf{N}_{B s} \mathbf{N}_{B s}^{\prime}\right)\left(\frac{1}{\sqrt{v_{1}}} \mathbf{1}_{v_{1}} \otimes \boldsymbol{y}_{i j}\right) \\
& =\sum_{s=1}^{t}\left(\mathbf{N}_{A s} \mathbf{N}_{A s}^{\prime} \frac{1}{\sqrt{v_{1}}} \mathbf{1}_{v_{1}}\right) \otimes\left(\mathbf{N}_{B s} \mathbf{N}_{B s}^{\prime} \boldsymbol{y}_{i j}\right) \\
& =\alpha_{1} k_{1}\left(k_{2}-q_{21}\right)\left(\frac{1}{\sqrt{v_{1}}} \mathbf{1}_{v_{1}} \otimes \boldsymbol{y}_{i j}\right) .
\end{aligned}
$$

The corresponding eigenvalue is $\alpha_{1} k_{1}\left(k_{2}-q_{21}\right)$.

For (6), we have

$$
\begin{aligned}
\mathbf{N}_{1} \mathbf{N}_{1}^{\prime}\left(\boldsymbol{x}_{i j} \otimes \boldsymbol{y}_{i j^{\prime}}\right) & =\sum_{s=1}^{t}\left(\mathbf{N}_{A s} \mathbf{N}_{A s}^{\prime} \otimes \mathbf{N}_{B s} \mathbf{N}_{B s}^{\prime}\right)\left(\boldsymbol{x}_{i j} \otimes \boldsymbol{y}_{i j^{\prime}}\right) \\
& =\sum_{s=1}^{t}\left(\mathbf{N}_{A s} \mathbf{N}_{A s}^{\prime} \boldsymbol{x}_{i j}\right) \otimes\left(\mathbf{N}_{B s} \mathbf{N}_{B s}^{\prime} \boldsymbol{y}_{i j^{\prime}}\right) \\
& =\left(k_{1}-q_{11}\right)\left(k_{2}-q_{21}\right)\left(\boldsymbol{x}_{i j} \otimes \boldsymbol{y}_{i j^{\prime}}\right) .
\end{aligned}
$$

The corresponding eigenvalue is $\left(k_{1}-q_{11}\right)\left(k_{2}-q_{21}\right)$.

For (3), (5) and (7)-(10), obviously, the vectors are the eigenvectors of $\mathbf{N}_{1} \mathbf{N}_{1}^{\prime}$ and the corresponding eigenvalue is 0 .

Similarly, from (8) and (9), the vectors of (1)-(10) are the eigenvectors of $\mathbf{N}_{2} \mathbf{N}_{2}^{\prime}$ and $\mathbf{N}_{3} \mathbf{N}_{3}^{\prime}$ and the corresponding eigenvalues with respect to $\mathbf{N}_{2} \mathbf{N}_{2}^{\prime}$ are $t \alpha_{1} \alpha_{2} k_{2}$ for (1)-(3), $\alpha_{1}\left(k_{2}-q_{21}\right)$ for (4) and (6)-(8), and 0 for (5), (9) and (10), and the corresponding eigenvalues with respect to $\mathbf{N}_{3} \mathbf{N}_{3}^{\prime}$ are $t \alpha_{1} k_{1} \alpha_{2}$ for (1), (4) and (5), ( $\left.k_{1}-q_{11}\right) \alpha_{2}$ for (2), (6), (7) and (9), and 0 for (3), (8) and (10).

The above argument is summarized in Table 3. 
Table 3. Eigenvalues and common eigenvectors

\begin{tabular}{|c|c|c|c|}
\hline \multicolumn{3}{|c|}{ Eigenvalues } & \multirow{2}{*}{$\begin{array}{c}\text { Common } \\
\text { eigenvectors }\end{array}$} \\
\hline $\mathbf{N}_{1} \mathbf{N}_{1}^{\prime}$ & $\mathbf{N}_{2} \mathbf{N}_{2}^{\prime}$ & $\mathbf{N}_{3} \mathbf{N}_{3}^{\prime}$ & \\
\hline$t \alpha_{1} k_{1} \alpha_{2} k_{2}$ & $t \alpha_{1} \alpha_{2} k_{2}$ & $t \alpha_{1} k_{1} \alpha_{2}$ & (1) \\
\hline$\left(k_{1}-q_{11}\right) \alpha_{2} k_{2}$ & $t \alpha_{1} \alpha_{2} k_{2}$ & $\left(k_{1}-q_{11}\right) \alpha_{2}$ & (2) \\
\hline 0 & $t \alpha_{1} \alpha_{2} k_{2}$ & 0 & (3) \\
\hline$\alpha_{1} k_{1}\left(k_{2}-q_{21}\right)$ & $\alpha_{1}\left(k_{2}-q_{21}\right)$ & $t \alpha_{1} k_{1} \alpha_{2}$ & (4) \\
\hline 0 & 0 & $t \alpha_{1} k_{1} \alpha_{2}$ & (5) \\
\hline$\left(k_{1}-q_{11}\right)\left(k_{2}-q_{21}\right)$ & $\alpha_{1}\left(k_{2}-q_{21}\right)$ & $\left(k_{1}-q_{11}\right) \alpha_{2}$ & (6) \\
\hline 0 & $\alpha_{1}\left(k_{2}-q_{21}\right)$ & $\left(k_{1}-q_{11}\right) \alpha_{2}$ & $(7)$ \\
\hline 0 & $\alpha_{1}\left(k_{2}-q_{21}\right)$ & 0 & (8) \\
\hline 0 & 0 & $\left(k_{1}-q_{11}\right) \alpha_{2}$ & (9) \\
\hline 0 & 0 & 0 & (10) \\
\hline
\end{tabular}

The vectors of (1)-(10) are also the common eigenvectors of the stratum information matrices $\mathbf{A}_{1}, \mathbf{A}_{2}, \mathbf{A}_{3}$ and $\mathbf{A}_{4}$. By use of (1) and Table 3, we give the stratum efficiency factors for the ISBD $\tilde{\mathcal{D}}$ in Table 4.

Table 4. Stratum efficiency factors for the ISBD $\tilde{\mathcal{D}}$

\begin{tabular}{cccccc}
\hline Type of & Number of & \multicolumn{5}{c}{ Strata } \\
\cline { 3 - 6 } contrasts & contrasts & I & II & III & IV \\
\hline$A$ & $t m_{1}$ & $\omega_{1}$ & $1-\omega_{1}$ & 0 & 0 \\
& $n_{1}$ & 0 & 1 & 0 & 0 \\
\hline$B$ & $t m_{2}$ & $\omega_{2}$ & 0 & $1-\omega_{2}$ & 0 \\
& $n_{2}$ & 0 & 0 & 1 & 0 \\
\hline$A \times B$ & $t m_{1} m_{2}$ & $t \omega_{1} \omega_{2}$ & $\omega_{2}\left(1-t \omega_{1}\right)$ & $\omega_{1}\left(1-t \omega_{2}\right)$ & $1-\omega_{1}-\omega_{2}+t \omega_{1} \omega_{2}$ \\
& $t(t-1) m_{1} m_{2}$ & 0 & $\omega_{2}$ & $\omega_{1}$ & $1-\omega_{1}-\omega_{2}$ \\
& $t n_{1} m_{2}$ & 0 & $\omega_{2}$ & 0 & $1-\omega_{2}$ \\
& $t m_{1} n_{2}$ & 0 & 0 & $\omega_{1}$ & $1-\omega_{1}$ \\
& $n_{1} n_{2}$ & 0 & 0 & 0 & 1 \\
\hline
\end{tabular}

Here $\omega_{1}=\left(k_{1}-q_{11}\right) /\left(t \alpha_{1} k_{1}\right)$ and $\omega_{2}=\left(k_{2}-q_{21}\right) /\left(t \alpha_{2} k_{2}\right), A$ and $B$ denote the basic contrasts among the main effects of row and column treatments and $A \times B$ denotes the basic contrasts among the interaction effects. The eigenvectors of (2)-(3), (4)-(5) and (6)-(10) define the basic contrasts $A$, $B$ and $A \times B$ respectively. We use Table 4 to improve the estimators for the basic contrasts of the treatment effects combining the estimators obtained from the strata I (inter-block stratum), II (inter-row, within blocks, stratum), III (inter-column, within blocks, stratum) and IV (inter-plot stra- 
tum). This procedure was proposed by Nelder (1965a, 1965b) and Houtman and Speed (1983). In particular, we see that some basic contrasts of $A, B$ and $A \times B$ are estimable with full efficiency. For a special case, where the affine $\alpha$-resolvable designs are square lattice designs for row and column treatments, the stratum efficiency factors are given in Mejza et al. (2009).

Example 2. For the ISBD $\tilde{\mathcal{D}}$ given in Example 1, $m_{1}=2, n_{1}=4, m_{2}=3$, $n_{2}=9, \omega_{1}=1 / 8$ and $\omega_{2}=1 / 18$. Thus, by use of Table 4 , the stratum efficiency factors can be calculated as in the following table:

Table 5. Stratum efficiency factors for Example 1

\begin{tabular}{cccccc}
\hline Type of & Number of & \multicolumn{4}{c}{ Strata } \\
\cline { 3 - 6 } contrasts & contrasts & I & II & III & IV \\
\hline$A$ & 4 & $1 / 8$ & $7 / 8$ & 0 & 0 \\
& 4 & 0 & 1 & 0 & 0 \\
\hline$B$ & 6 & $1 / 18$ & 0 & $17 / 18$ & 0 \\
& 9 & 0 & 0 & 1 & 0 \\
\hline$A \times B$ & 12 & $1 / 72$ & $1 / 24$ & $1 / 9$ & $5 / 6$ \\
& 12 & 0 & $1 / 18$ & $1 / 8$ & $59 / 72$ \\
& 24 & 0 & $1 / 18$ & 0 & $17 / 18$ \\
& 36 & 0 & 0 & $1 / 8$ & $7 / 8$ \\
& 36 & 0 & 0 & 0 & 1 \\
\hline
\end{tabular}

\section{Acknowledgements}

The research was partially supported by JSPS Grant-in-Aid for Scientific Research (B) 15H03636 and (C) 16K00053.

\section{REFERENCES}

Bailey R.A., Monod H., Morgan, J.P. (1995): Construction and optimality of affineresolvable designs, Biometrika 82(1): 187-200.

Caliński T., Kageyama S. (2003) Block Designs: A Randomization Approach, Volume II: Design, Lecture Notes in Statistics 170, New York, Springer.

Hering F., Mejza S. (1997): Incomplete split-block designs. Biometrical Journal 39: $227-238$.

Hering F., Mejza S. (2002): An incomplete split-block design generated by GDPBIBD(2)s. Journal of Statistical Planning and Inference 106: 125-134.

Houtman A.M., Speed T.P. (1983): Balance in designed experiments with orthogonal block structure. The Annals of Statistics 11: 1069-1085.

Kadowaki S., Kageyama S. (2009): Existence of affine $\alpha$-resolvable PBIB designs with some constructions. Hiroshima Mathematical Journal 39: 293-326. 
Kageyama S. (1973): On $\mu$-resolvable and affine $\mu$-resolvable balanced incomplete block designs. The Annals of Statistics 1: 195-203.

Khatri C.G., Rao C.R. (1968): Solutions to some functional equations and their applications to characterization of probability distributions. Sankhyā A 30: 167-180.

Kuriki S., Mejza I., Jimbo M., Mejza S., Ozawa K. (2005): Resolvable semibalanced incomplete split-block designs. Metrika 61: 9-16.

Mejza I. (1987): A note on the analysis of split-block designs. In: Proceedings of the Statistical Data Analysis '87. Bulgarian Academy of Sciences: 128-132.

Mejza I. (1998): Characterisation of certain split-block designs with a control. Biometrical Journal 40: 627-639.

Mejza I., Kuriki S., Mejza S. (2001): Balanced square lattice designs in split-block designs. Colloquium Biometryczne 31: 97-103.

Mejza I., Mejza S., Kuriki S. (2009): Square lattice designs in incomplete splitblock designs. Journal of Statistics and Applications 4: 327-338.

Mejza S. (1992): On some aspects of general balance in designed experiments. Statistica 52: 263-278.

Nelder J.A. (1965a): The analysis of randomized experiments with orthogonal block structure. I. Block structure and the null analysis of variance. Proceedings of the Royal Society. London A 283, 147-162.

Nelder J.A. (1965b): The analysis of randomized experiments with orthogonal block structure. II. Treatment structure and the general analysis of variance. Proceedings of the Royal Society. London A 283: 163-178.

Ozawa K., Jimbo M., Kageyama S., Mejza S. (2002a): Optimality and constructions of incomplete split-block designs. Journal of Statistical Planning and Inference 106: 135-157.

Ozawa K., Jimbo M., Kageyama S., Mejza S. (2002b): Optimality and efficiency of incomplete split-block designs. Metrika 56: 1-18.

Ozawa K., Kuriki S., Yumiba H. Jimbo M. (2000): Balanced and semi-balanced incomplete split-block designs. Congressus Numerantium 145: 193-201.

Pearce S.C., Caliński T., Marshall T.F. de C. (1974): The basic contrasts of an experimental design with special reference to the analysis of data. Biometrika 61: 449-460. 